\title{
Heavier alkali-metal monosulfides (KS, RbS, CsS, and FrS) and their cations
}

\author{
Edmond P. F. Lee ${ }^{\text {a) }}$ \\ School of Chemistry, University of Southampton, Highfield, Southampton SO17 1BJ United Kingdom \\ and Department of Applied Biology and Chemical Technology, Hong Kong Polytechnic University, \\ Hung Hom, Hong Kong \\ Timothy G. Wright ${ }^{\text {b) }}$ \\ School of Chemistry, University of Nottingham, University Park, Nottingham NG7 2RD, United Kingdom
}

(Received 17 June 2005; accepted 2 August 2005; published online 11 October 2005)

\begin{abstract}
The heavier alkali-metal monosulfides (KS, RbS, CsS, and FrS) have been studied by high-level $a b$ initio calculations. The RCCSD(T) method has been employed, combined with large flexible valence basis sets. All-electron basis sets are used for potassium and sulfur, with effective core potentials being used for the other metals, describing the core electrons. Potential-energy curves are calculated for the lowest two neutral and cationic states: all neutral monosulfide species have a ${ }^{2} \Pi$ ground state, in contrast with the alkali-metal monoxide species, which undergo a change in the electronic ground state from ${ }^{2} \Pi$ to ${ }^{2} \Sigma^{+}$as the group is descended. In the cases of KS, RbS, and CsS, spin-orbit curves are also calculated. We also calculate potential-energy curves for the lowest ${ }^{3} \Sigma^{-}$ and ${ }^{3} \Pi$ states of the cations. From the potential-energy curves, spectroscopic constants are derived, and for KS the spectroscopic results are compared to experimental spectroscopic values. Ionization energies, dissociation energies, and heats of formation are also calculated; for KS, we explore the effects of relativity and basis set extrapolation on these values. (c) 2005 American Institute of Physics. [DOI: 10.1063/1.2042450]
\end{abstract}

\section{INTRODUCTION}

The alkali-metal monoxides (MO) are noteworthy for exhibiting a change in the electronic ground state on descending the group: LiO (Refs. 1 and 2) and $\mathrm{NaO}$ (Ref. 3) have ${ }^{2} \Pi$ ground states and $\mathrm{RbO}$ (Ref. 4) and $\mathrm{CsO}$ (Ref. 5) have ${ }^{2} \Sigma^{+}$ground states, as confirmed by microwave spectroscopy. An explanation for this change in ground state has been put forward by Allison and Goddard ${ }^{6}$ and Allison et al.: $:^{7}$ basically, the long-range attractive electrostatic terms favor the ${ }^{2} \Pi$ state, whereas the ${ }^{2} \Sigma^{+}$state is favored at a shorter range, owing to the Pauli repulsion term. Which state is the lowest is a result of the balance of these two effects as a function of the internuclear separation and, as discussed in Refs. 6 and 7, this leads to the ${ }^{2} \Sigma^{+}$being more favored as the group is descended.

Of course, the crossover has to occur somewhere, and $\mathrm{KO}$ has proven to be rather a contentious molecule from this point of view. Rather than review all of the literature, we refer the reader to the introductions of the papers cited below, and simply note that there has been a range of both experimental and theoretical studies on $\mathrm{KO}$, with a significant number concluding a ${ }^{2} \Pi$ ground state, but also a significant number concluding a ${ }^{2} \Sigma^{+}$ground state. Usually microwave experiments are quite definitive about the symmetry of the ground state of a diatomic molecule, and of note is that Hirota ${ }^{8}$ has discussed the microwave spectra of $\mathrm{KO}$ and noted therein that he and others ${ }^{9}$ had recorded a microwave

\footnotetext{
${ }^{a}$ Electronic mail: e.p.lee@soton.ac.uk

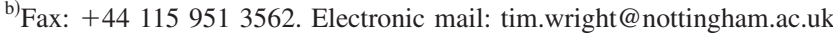

spectrum of both the ${ }^{2} \Pi$ and ${ }^{2} \Sigma^{+}$states of $\mathrm{KO}$ and concluded that the ground state was ${ }^{2} \Pi$. This is in contrast to a highlevel $a b$ initio calculation ${ }^{10}$ by Bauschlicher et al. who concluded that there was a very little chance that any further calculations would conclude anything other than that the ground state of $\mathrm{KO}$ was ${ }^{2} \Sigma^{+}$. In 2002, we published a paper ${ }^{11}$ that calculated the spin-orbit states of KO (in Ref. 10, spinorbit coupling was considered, but only to see if it reversed the ordering of states, which it did not). The ground electronic state of $\mathrm{KO}$ is an $\Omega=1 / 2$ state, designated by $X \frac{1}{2}$, which is essentially a ${ }^{2} \Sigma_{1 / 2}^{+}$state to a short range, but at $\sim 2.3 \AA$, after an avoided crossing between the ${ }^{2} \Sigma_{1 / 2}^{+}$state and the ${ }^{2} \Pi_{1 / 2}$ state, it becomes the latter. Hence the ground state of $\mathrm{KO}$ has ${ }^{2} \Sigma^{+}$character to a short range and ${ }^{2} \Pi_{1 / 2}$ character to a long range. The presence of the avoided crossing gives rise to a "shelf" between 2.3 and $2.4 \AA$, and the $v=0$ level was calculated to lie just below this shelf: there is therefore a mixed character for this state, which might be the cause of the apparently contradictory results of previous experiments. Hirota ${ }^{8}$ noted, in particular, that the analysis of the microwave spectra demonstrated some difficulties.

The alkali-metal monosulfides (MS) have also been the subject of some attention, but nowhere near as much as the monoxides. The lighter MS species have been studied by microwave spectroscopy, with $\mathrm{LiS},{ }^{12} \mathrm{NaS},{ }^{13}$ and $\mathrm{KS}^{14}$, all being found to have ${ }^{2} \Pi_{i}$ ground states. It was commented in Ref. 14 that the change in the ground electronic state is caused by the Pauli repulsion, and that hence the ${ }^{2} \Sigma^{+}$state ought to be favored earlier in the alkali-metal series for MS than is the case for MO; however, we note here that both the 
attractive and repulsive terms will change on going from $\mathrm{MO}$ to MS, and so it is perhaps not quite so obvious what the implications would be for the ${ }^{2} \Sigma^{+}-2 \Pi$ crossover. In addition, we note that the authors of Ref. 14 had commented that no calculations had been carried out on these sulfides, whereas, in fact, Partridge et al. had published such a study ten years earlier ${ }^{15}$ for the LiS-RbS species, and had concluded that they all had ${ }^{2} \Pi$ ground states; it was also commented therein that $\mathrm{CsS}$ was likely to have a ${ }^{2} \Pi$ ground state. Consequently, therefore, both experiment and theory agree with respect to the ground-state symmetries of LiS-KS.

The purpose of the present paper is firstly to report highquality calculations for the heavier MS species, including $\mathrm{CsS}$ and $\mathrm{FrS}$ in order to deduce the ground electronic states of these species. This work follows on from our previous work ${ }^{16}$ on $\mathrm{LiS} / \mathrm{LiS}^{+}$and $\mathrm{NaS} / \mathrm{NaS}^{+}$. Also, the effects of spin-orbit coupling will also be examined for the KS-CsS species (it seems unlikely that FrS will be observed, and given the lack of all-electron basis sets for this species, required for the spin-orbit calculations, we do not perform spin-orbit calculations for this species). In addition, we calculate spectroscopic parameters for these species, with and without spin-orbit coupling, and in the case of KS we compare our values to the available experimental data.

Finally, we also perform calculations on the cations in order to obtain ionization energies.

\section{THEORETICAL DETAILS}

$\operatorname{RCCSD}(\mathrm{T})$ calculations were carried out for all species making use of the MOLPRO suite of programs. For S, the standard aug-cc-pV5Z basis set was used: all functions were used in the $\mathrm{KS} / \mathrm{KS}^{+}$calculations, but the $h$ functions were omitted for the other calculations. For K, a basis set has been constructed previously that is of a similar quality to aug-cc$\mathrm{pV} 5 \mathrm{Z}$, and the reader is referred to Ref. 11. For the heavier alkali metals, effective core potentials (ECPs) were used. It is important to note that these species are largely ionic and that the outermost $n s$ orbital is absent in $\mathrm{M}^{+}$, consequently the $(n-1) s$ and $(n-1) p$ orbitals are effectively the valence electrons and considered as such herein. Consequently, we select ECPs which only describe the innermost electrons. The valence region is described by a large, flexible basis set, which has been described previously. We summarize the basis sets below, but the full details can be obtained from the given references.

(a) For K, the $[12 s 12 p 6 d 4 f 3 g 2 h]$ basis set has been described in Ref. 11.

(b) For Rb, the ECP28MWB ECP was employed, with the valence region being described by $[10 s 8 p 5 d 4 f 3 g]$ functions, described in full in Ref. 17.

(c) For Cs, the ECP46MWB ECP was employed, with the valence region being described by $[10 s 8 p 5 d 4 f 3 g]$ functions, described in full in Ref. 17.

(d) For Fr, the CRENBL78 ECP was employed, with the valence region being described by $[11 s 10 p 5 d 4 f 3 g]$ functions, described in full in Ref. 17.

To obtain the potential-energy curves, energies were cal- culated at a range of bond lengths covering short to moderately long range, such that the crossing between the ${ }^{2} \Sigma^{+}$and the ${ }^{2} \Pi$ states was covered. Each energy point was corrected for basis set superposition error (BSSE) by making use of the full counterpoise (CP) correction. The moieties used in the correction were $\mathrm{M}^{+}$and $\mathrm{S}^{-}$.

\section{A. Spin-orbit calculations}

For the spin-orbit calculations, complete active space self-consistent-field (CASSCF) calculations were carried out employing uncontracted basis sets, specified below. In these calculations, the $3 s$ and $3 p$ orbitals of sulfur, and the $(n$ $-1) s,(n-1) p$, and $n s$ orbitals of $\mathrm{M}^{+}$(where the $n s$ electron of $M$ is lost in forming $\mathrm{M}^{+}$) are used in the active space, and the counterpoise-corrected RCCSD(T) energies were used as the diagonal elements of the spin-orbit matrix. Spin-orbit coupling employing the Breit-Pauli operator was calculated between the three lowest $\Omega$ states, which includes the interaction between the $\Omega=1 / 2$ states: this leads to avoided crossings between the $\Omega=1 / 2$ states.

The basis sets used in these calculations were allelectron, consisted of $s, p$, and $d$ functions only, were used uncontracted, and are all described in full on the Gaussian basis set order form (GBSOF) website. ${ }^{18}$ They were the Partridge 2 basis set ${ }^{19}$ for potassium, the Partridge 1 basis set for rubidium, ${ }^{20}$ and the Huzinaga Midi basis set for cesium. ${ }^{21}$ In all cases the aug-cc-pVTZ basis set was used for sulfur, again employing the $s, p$, and $d$ functions only.

\section{B. Spectroscopy}

From the interaction potential-energy functions, equilibrium internuclear separations, dissociation energies, and rovibrational energy levels were obtained using LeRoy's LEVEL program. ${ }^{22}$ In each case, the most abundant isotopomer was considered.

\section{RESULTS AND DISCUSSION}

\section{A. KS}

The KS radical has been studied by Xin and Ziurys, ${ }^{14}$ who produced the radical by mixing $\mathrm{K}(g)$ with $\mathrm{CS}_{2}(g)$ in argon, and then discharging the mixture. Spin-orbit components assigned to the ${ }^{2} \Pi_{3 / 2}$ and ${ }^{2} \Pi_{1 / 2}$ states were observed, with the observed transitions being between rather high $J$ levels $\left(J^{\prime \prime}=33.5-55.5\right)$. The much smaller lambda-doubling splitting in the ${ }^{2} \Pi_{3 / 2}$ state confirmed that the state was indeed inverted; and the detection of both spin-orbit states confirms the identity as ${ }^{2} \Pi_{i}$. We note that the alkali-metal monoxides form in the ${ }^{2} \Sigma^{+}$excited state when alkali-metal atoms are reacted with $\mathrm{N}_{2} \mathrm{O}$ or $\mathrm{O}_{3},{ }^{23,24}$ and so of course, it is possible that an electronically excited state of KS is produced, but this will likely have decayed faster than the rotational transitions. By fitting the observed rotational transition frequencies for $\mathrm{KS}$, to a Hamiltonian involving rotational, spin-orbit, and centrifugal distortion terms of each of these, together with the lambda doubling, good fits were obtained by Xin and 


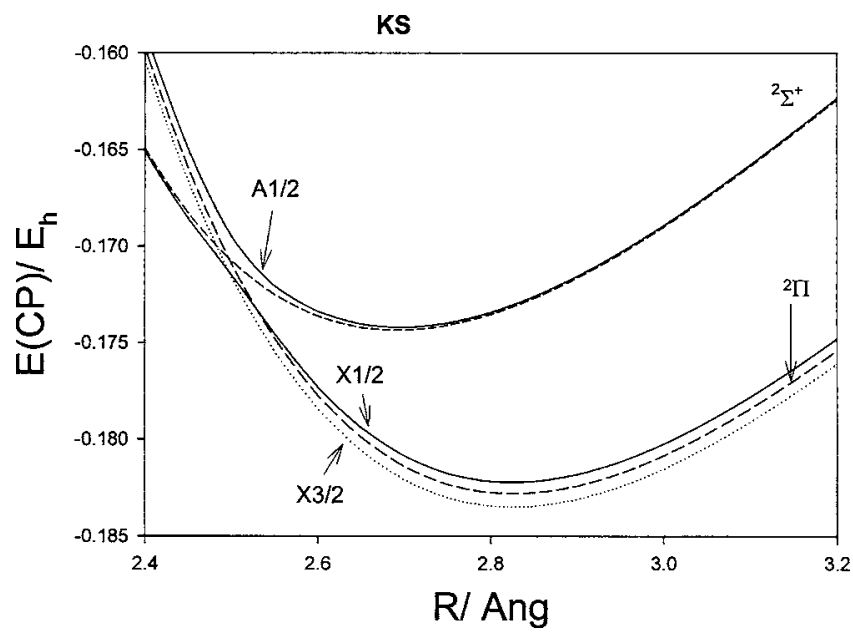

FIG. 1. Calculated potential-energy curves for KS in the $R_{e}$ region. The ${ }^{2} \Sigma^{+}$ and ${ }^{2} \Pi$ curves (dashed lines) are calculated in the absence of spin-orbit coupling; the other three curves arise from these two states when spin-orbit coupling occurs. The two $\Omega=1 / 2$ states undergo an avoided crossing. See text for details.

Ziurys $^{14}$ for a number of spectroscopic parameters. It was noted that the spin-orbit terms were rather insensitive to the data, owing to the high $J$ number sampled.

As noted above, we calculated ${ }^{2} \Pi$ and ${ }^{2} \Sigma^{+}$curves, and then investigated the effect of spin-orbit coupling on the shapes of these curves. This is presented graphically in Fig. 1. To first order, the ${ }^{2} \Pi$ state splits into $\Omega=3 / 2$ and $1 / 2$ spin-orbit states; the $\Omega=3 / 2$ state is unaffected by any further interactions at this level of approximation, and so the calculated spectroscopic parameters for this state (represented by $X \frac{3}{2}$ ) are almost identical to those of the ${ }^{2} \Pi$ state. The $\Omega=1 / 2$ component of the $\Pi$ state can interact with the ${ }^{2} \Sigma^{+}$state, which also has $\Omega=1 / 2$. Since the ${ }^{2} \Pi$ and the ${ }^{2} \Sigma^{+}$ curves cross, then there has to be an avoided crossing between the two $\Omega=1 / 2$ states. We therefore label these states as $X \frac{1}{2}$ and $A \frac{1}{2}$. The $X \frac{1}{2}$ state correlates with the ${ }^{2} \Pi_{1 / 2}$ state to long $\mathrm{R}$, but to the ${ }^{2} \Sigma^{+}$state to short $\mathrm{R}$; similarly, the $A \frac{1}{2}$ state correlates to the ${ }^{2} \Sigma^{+}$state to long $R$, but to the ${ }^{2} \Pi_{1 / 2}$ state to short $\mathrm{R}$. The avoided crossing occurs fairly high above the minimum of the ${ }^{2} \Pi$ state, and so the calculated spectroscopic parameters in the vicinity of the minimum are similar to the unperturbed ${ }^{2} \Pi$ state for the $X \frac{1}{2}$ state; however, the crossing is much closer to the minimum of the ${ }^{2} \Sigma^{+}$state, and leads to a steepening of the curve to short $\mathrm{R}$. This leads to the $A \frac{1}{2}$ state having a slightly higher vibrational frequency than the ${ }^{2} \Sigma^{+}$state, and also leads to this state having a negative anharmonicity.

In the preliminary report of the microwave work on $\mathrm{KO}$, Hirota $^{8}$ noted that observed transitions were assigned as belonging to both the ${ }^{2} \Pi$ state and the ${ }^{2} \Sigma^{+}$state, and consequently a simultaneous fit to all three spin-orbit states was attempted. However, it was noted that the fit to higher rotational energy levels was unsatisfactory. That high rotational energy levels were those observed in the KS experiments, coupled with the possibility that it might be necessary to fit both all three spin-orbit states simultaneously, means that a direct comparison between the experimental data and the calculated ones below should be viewed with a small pinch of caution. That said, the errors in the experimentally derived parameters seem to be very small, which is perhaps related to the fact that the crossing is high enough up the repulsive wall of the ${ }^{2} \Pi$ state, that the spectroscopies of the ${ }^{2} \Pi_{3 / 2}$ and ${ }^{2} \Pi_{1 / 2}$ states are very similar.

In Table I, we present the spectroscopic data obtained from our RCCSD(T) curves. We compare our values to the previous experimental and theoretical values, considering the ${ }^{2} \Pi$ state first. Our $B_{0}$ values agree very well with the experimental value. No $B_{e}$ value (and hence $R_{e}$ value) was obtained from the experiment, as only one vibrational level was observed. Estimating an $R_{0}$ value from our $B_{0}$ value gives $R_{0}$ $=2.828 \AA$, close to the value of $2.817 \AA$ estimated using the experimental $B_{0}$ value. Our calculated $R_{e}$ value is also in fairly good agreement with that reported in Ref. 15 , with the singles and doubles configuration-interaction (SDCI) value being a little longer, and the estimated $R_{e}$ value (adjusted therein from a knowledge of the performance of the methods employed) is slightly shorter. We also note that our value for the first centrifugal distortion coefficient, $D_{0}$, was calculated to be $1.075 \times 10^{-7} \mathrm{~cm}^{-1}$, which is very close to the experimental value ${ }^{14}$ of $1.088 \times 10^{-7} \mathrm{~cm}^{-1}$.

It is also possible to estimate the vibrational frequency from the experimental rotational data using $\omega^{2}=4 B^{3} / D$, yielding a value of $255 \mathrm{~cm}^{-1}$, which is in excellent agree-

TABLE I. Calculated spectroscopic constants for ${ }^{39} \mathrm{~K}^{32} \mathrm{~S}$.

\begin{tabular}{lccccccc}
\hline \hline State & $\begin{array}{c}R_{e} \\
(\AA)\end{array}$ & $\begin{array}{c}B_{0} \\
\left(\mathrm{~cm}^{-1}\right)\end{array}$ & $\alpha$ & $\begin{array}{c}\nu_{0-1} \\
\left(\mathrm{~cm}^{-1}\right)\end{array}$ & $\begin{array}{c}\omega_{e} \\
\left(\mathrm{~cm}^{-1}\right)\end{array}$ & $\begin{array}{c}\omega_{e} x_{e} \\
\left(\mathrm{~cm}^{-1}\right)\end{array}$ & \\
\hline${ }^{2} \Pi$ & 2.825 & 0.1199 & $7.00 \times 10^{-4}$ & 253.4 & 255.5 & 1.02 & \\
$X^{\frac{3}{2}}$ & 2.824 & 0.1200 & $6.78 \times 10^{-4}$ & 252.7 & 254.8 & 1.05 & \\
$X_{2}^{1}$ & 2.825 & 0.1199 & $7.00 \times 10^{-4}$ & 253.4 & 255.5 & 1.03 & \\
${ }^{2} \Sigma^{+}$ & 2.692 & 0.1321 & $8.50 \times 10^{-4}$ & 255.2 & 257.4 & 1.11 & \\
$A_{2} \frac{1}{2}$ & 2.695 & 0.1316 & $1.25 \times 10^{-3}$ & 261.1 & 260.2 & -0.46 & \\
${ }^{2} \Pi_{i}$ & 2.817 & 0.1210 & & & 255 & & Expt. $^{\text {a }}$ \\
${ }^{2} \Pi$ & 2.842 & & & & 240 & & Previous $^{\text {Theor. }}$ \\
${ }^{2} \Sigma^{+}$ & 2.717 & & & & 257 & & b $^{+}$ \\
\hline \hline
\end{tabular}

a(Sub)-millimeter rotational spectroscopy (Ref. 14). Bond length is $R_{0}$, calculated from the $B_{0}$ value. The harmonic vibrational frequency was calculated by us from the relation $D=4 B^{3} / \omega^{2}$, where we used the $B_{0}$ and $D_{0}$ parameters reported in this work.

${ }^{\mathrm{b}} \mathrm{SDCI}$ results from Ref. 15. An estimate of $2.81 \AA$ was made for the ${ }^{2} \Pi$ state of KS, based upon previous estimates of the reliability of the calculations. 
ment with the values reported in Table I for the ${ }^{2} \Pi$ state. The value of $240 \mathrm{~cm}^{-1}$ from Ref. 15 is slightly lower, but overall very good agreement is seen.

The spin-orbit splitting constant, $A$, was derived as $-300.2 \mathrm{~cm}^{-1}$ in Ref. 14. The calculated energy difference between the $v=0$ levels of the $X \frac{3}{2}$ and $X \frac{1}{2}$ states is $-278.0 \mathrm{~cm}^{-1}$, which is in reasonable agreement with the experimentally derived value; we note that the latter value has been derived from highly rotationally excited levels, and it was noted in Ref. 14 that $A$ was rather insensitive to the fit, and so we feel the agreement is reasonable. We also note that an unperturbed value for $A$ can be estimated from twice the energy difference between the $v=0$ levels of the $X_{2}^{\frac{3}{2}}$ and unperturbed ${ }^{2} \Pi$ state, giving a value of $-300.2 \mathrm{~cm}^{-1}$, this value is in perfect agreement with the experimental value, and suggests that caution is merited in comparing the calculated and experimental values in the absence of inclusion of the $A^{2} \Sigma^{+}$(or better, the $A \frac{1}{2}$ state) in the experimental analysis.

As noted above, the ${ }^{2} \Sigma^{+}$state was not observed experimentally, however, Partridge et al. calculated ${ }^{15} R_{e}=2.717 \AA$ and $\omega_{e}=257 \mathrm{~cm}^{-1}$, both of which are close to the values we report herein.

The energy gap, $T_{0}$, between the ${ }^{2} \Pi$ and the ${ }^{2} \Sigma^{+}$states was estimated from the observed lambda-doubling constants as $1900 \mathrm{~cm}^{-1}$ for KS. This estimate was based upon the assumption of the pure precession hypothesis, described by van Vleck ${ }^{25}$ and Mulliken and Christie. ${ }^{26}$ The validity of the assumption relies on the $\Pi$ and $\Sigma$ states behaving as if the electronic configuration is identical except that one electron has $\lambda=0$ and the other has $\lambda=1$. For KS, the configurations are $\sigma^{1} \pi^{4}$ and $\sigma^{2} \pi^{3}$, and so the hypothesis is clearly an approximation here. Our calculated $T_{0}$ value, in the absence of spin-orbit coupling, is $1855 \mathrm{~cm}^{-1}$ showing that despite the differences in the electronic configurations, the pure precession hypothesis appears to work well here (vide infra). This is in contrast to LiS and $\mathrm{NaS}$, where the pure precession estimate of the corresponding $T_{0}$ value was significantly lower than the calculated values ${ }^{16}$ - good agreement was, however, observed between our previous calculated values, and those ${ }^{15}$ of Partridge et al., confirming that it is the experimental estimates that are at fault. We note that for KS, the previously calculated $T_{e}$ value ${ }^{15}$ of $1834 \mathrm{~cm}^{-1}$ is also very close to our $T_{e}$ value of $1854 \mathrm{~cm}^{-1}$. We now examine other factors which may affect our calculated ${ }^{2} \Sigma^{+}-{ }^{2} \Pi$ separations.

In order to examine the role of relativistic effects on the KS molecule (note that for the heavier molecules, relativistic effects are incorporated, to some extent at least, into the ECP used), we included the mass-velocity and one-electron Darwin terms as implemented in the Cowan-Griffin operator, and also using the Douglas-Kroll one-electron integral approach. For these calculations, the $(24 s 16 p 6 d)$ functions, which underlie the contracted "aug-cc-pV5Z" basis set used herein, were used for potassium, with the $s, p$, and $d$ primitive functions of the standard aug-cc-pV5Z basis set being used for sulfur. The results from both of these approaches were rather similar, and led to the ${ }^{2} \Sigma^{+}-2 \Pi$ separation increasing by $17.5 \mathrm{~cm}^{-1}$.

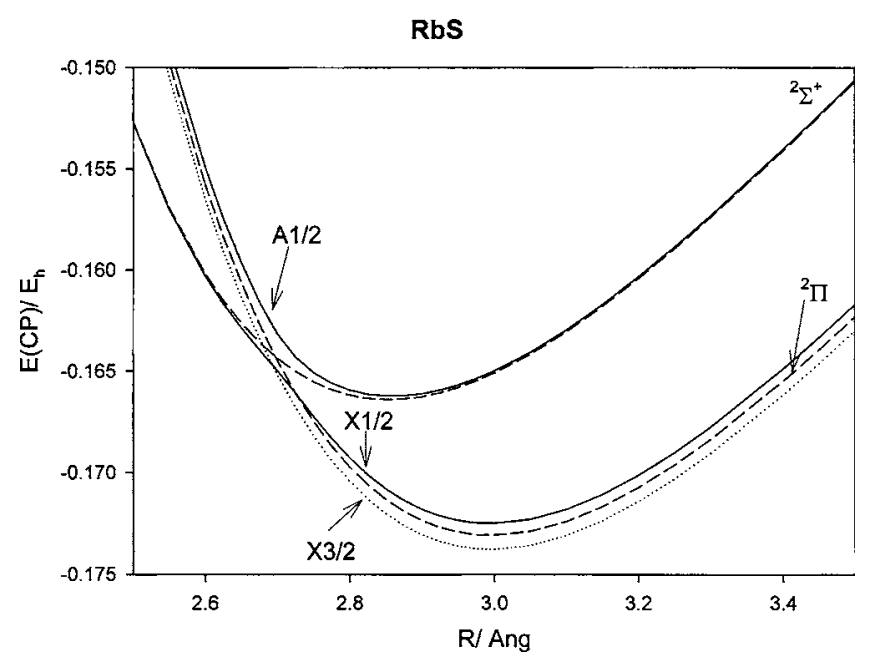

FIG. 2. Calculated potential-energy curves for $\mathrm{RbS}$ in the $R_{e}$ region. The ${ }^{2} \Sigma^{+}$and ${ }^{2} \Pi$ curves (dashed lines) are calculated in the absence of spin-orbit coupling; the other three curves arise from these two states when spin-orbit coupling occurs. The two $\Omega=1 / 2$ states undergo an avoided crossing. See text for details.

We also performed single-point aug-cc-pV6Z calculations (the sulfur basis set is the standard one, while the potassium one is basis set $D$ from Ref. 11), which lead to a $T_{e}$ value of $1841 \mathrm{~cm}^{-1}$, which is only $13 \mathrm{~cm}^{-1}$ lower than the aug-cc-pV5Z value. We extrapolate the $T_{e}$ value to $\infty$, using the formula ${ }^{27}$ of Helgaker et al. to yield an aug-cc-pVœZ value of $1823.1 \mathrm{~cm}^{-1}$. Correcting this for zero-point vibrational energy, we obtain $T_{0}=1824.6 \mathrm{~cm}^{-1}$, and adding on the relativistic correction gives a best value of $1842 \mathrm{~cm}^{-1}$. Note that this value refers to the separation of the unperturbed ${ }^{2} \Pi$ and ${ }^{2} \Sigma^{+}$states, and is still in reasonable agreement with the pure precession value. At the aug-cc-pV6Z level, we also calculated the BSSE for the ${ }^{2} \Pi$ state at the minimum, which amounts to $67 \mathrm{~cm}^{-1}\left(\mathrm{~K}^{+}\right)$and $16 \mathrm{~cm}^{-1}\left(\mathrm{~S}^{-}\right)$; for the ${ }^{2} \Sigma^{+}$state, the BSSE is the same for $\mathrm{K}^{+}$, and is $14 \mathrm{~cm}^{-1}$ for $\mathrm{S}^{-}$. The error in the ${ }^{2} \Sigma^{+}-2 \Pi$ separation arising from the BSSE is therefore only a few $\mathrm{cm}^{-1}$.

\section{B. RbS}

We present our calculated potential-energy curves for $\mathrm{RbS}$ in Fig. 2, with the calculated spectroscopic constants in Table II.

TABLE II. Calculated spectroscopic constants for ${ }^{85} \mathrm{Rb}^{32} \mathrm{~S}$.

\begin{tabular}{|c|c|c|c|c|c|c|c|}
\hline State & $\begin{array}{l}R_{e} \\
(\AA)\end{array}$ & $\begin{array}{c}B_{0} \\
\left(\mathrm{~cm}^{-1}\right)\end{array}$ & $\alpha$ & $\begin{array}{c}\nu_{0-1} \\
\left(\mathrm{~cm}^{-1}\right)\end{array}$ & $\begin{array}{c}\omega_{e} \\
\left(\mathrm{~cm}^{-1}\right)\end{array}$ & $\begin{array}{c}\omega_{e} x_{e} \\
\left(\mathrm{~cm}^{-1}\right)\end{array}$ & \\
\hline${ }^{2} \Pi$ & 2.996 & 0.0806 & $4.11 \times 10^{-4}$ & 209.6 & 211.0 & 0.738 & \\
\hline$X \frac{3}{2}$ & 2.996 & 0.0806 & $4.11 \times 10^{-4}$ & 209.5 & 211.0 & 0.726 & \\
\hline$X \frac{1}{2}$ & 2.995 & 0.0807 & $3.85 \times 10^{-4}$ & 208.6 & 210.3 & 0.084 & \\
\hline${ }^{2} \Sigma^{+}$ & 2.854 & 0.0889 & $4.88 \times 10^{-4}$ & 212.6 & 214.3 & 0.821 & \\
\hline$A \frac{1}{2}$ & 2.860 & 0.0883 & $9.14 \times 10^{-4}$ & 223.3 & 220.8 & -1.247 & \\
\hline${ }^{2} \Pi$ & 2.982 & & & & 210 & & Previous \\
\hline${ }^{2} \Sigma^{+}$ & 2.834 & & & & 206 & & Theor. $^{\text {a }}$ \\
\hline
\end{tabular}

${ }^{\mathrm{a}} \mathrm{SDCI}$ results from Ref. 15 . An estimate of $2.94 \AA$ was made for the ${ }^{2} \Pi$ state of $\mathrm{RbS}$, based upon previous estimates of the reliability of the calculations. 
CsS

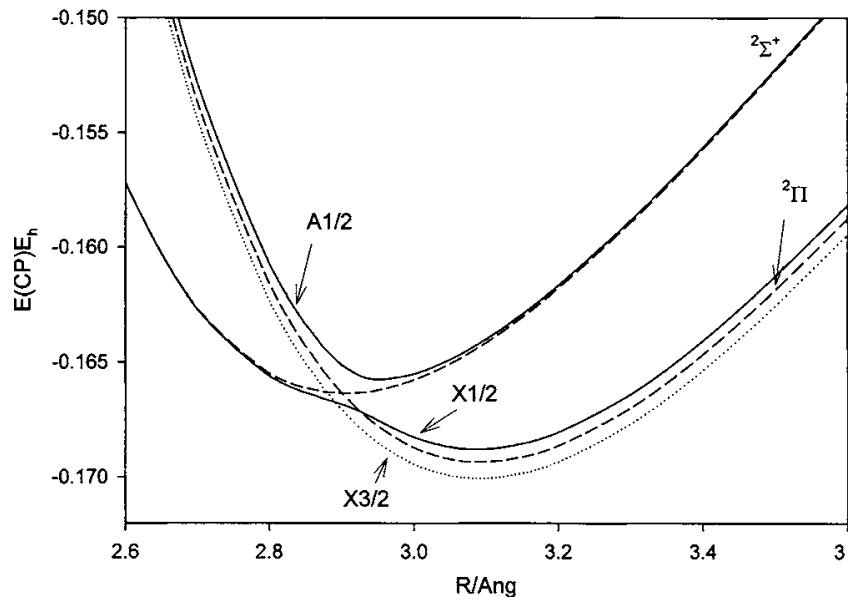

FIG. 3. Calculated potential-energy curves for CsS in the $R_{e}$ region. The ${ }^{2} \Sigma^{+}$ and ${ }^{2} \Pi$ curves (dashed lines) are calculated in the absence of spin-orbit coupling; the other three curves arise from these two states when spin-orbit coupling occurs. The two $\Omega=1 / 2$ states undergo an avoided crossing. See text for details.

There have been no experimental values reported for $\mathrm{RbS}$, but Partridge et al. calculated the spectroscopic constants for both the ${ }^{2} \Pi$ and ${ }^{2} \Sigma^{+}$states, in the absence of spinorbit coupling. ${ }^{15}$ Their values are in fairly good agreement with ours (see Table II), however, there is an anomaly in that the $\omega_{e}$ value for the ${ }^{2} \Sigma^{+}$state of $\mathrm{RbS}$ is calculated to be lower than that of the ${ }^{2} \Pi$ state, which is in contrast to their calculated values for $\mathrm{LiS}, \mathrm{NaS}$, and $\mathrm{KS}$; it is also contradictory to the ordering of our calculated values, without spinorbit coupling.

In Fig. 2, it may be seen that the ${ }^{2} \Sigma^{+}$state crosses the ${ }^{2} \Pi$ state lower down the repulsive wall than is the case for KS. This is reflected in slightly more pronounced differences between the calculated spectroscopic parameters of the ${ }^{2} \Sigma^{+}$ state and those of the $A \frac{1}{2}$ state.

Regarding the ${ }^{2} \Sigma^{+}{ }^{2} \Pi$ separation, we obtain $T_{e}\left(T_{0}\right)$ values of $1465(1467) \mathrm{cm}^{-1}$, which compare to the $T_{e}$ value reported in Ref. 15 of $1284 \mathrm{~cm}^{-1}$; the latter is somewhat lower than our value.

\section{CsS}

Our calculated potential-energy curves for $\mathrm{CsS}$ are given in Fig. 3; as may be seen, the crossing is lower down the repulsive wall than it was in the two previous cases, and in this case leads to significant deviations between the calculated spectroscopic parameters of the ${ }^{2} \Sigma^{+}$state and those of the $A \frac{1}{2}$ state. There are also more marked deviations between

TABLE III. Calculated spectroscopic constants for ${ }^{132} \mathrm{Cs}^{32} \mathrm{~S}$.

\begin{tabular}{ccccccc}
\hline \hline State & $\begin{array}{c}R_{e} \\
(\AA)\end{array}$ & $\begin{array}{c}B_{0} \\
\left(\mathrm{~cm}^{-1}\right)\end{array}$ & $\alpha$ & $\begin{array}{c}\nu_{0-1} \\
\left(\mathrm{~cm}^{-1}\right)\end{array}$ & $\begin{array}{c}\omega_{e} \\
\left(\mathrm{~cm}^{-1}\right)\end{array}$ & $\begin{array}{c}\omega_{e} x_{e} \\
\left(\mathrm{~cm}^{-1}\right)\end{array}$ \\
\hline${ }^{2} \Pi$ & 3.092 & 0.0683 & $3.16 \times 10^{-4}$ & 194.5 & 195.8 & 0.639 \\
$X \frac{3}{2}$ & 3.091 & 0.0683 & $3.15 \times 10^{-4}$ & 194.5 & 195.7 & 0.595 \\
$X \frac{1}{2}$ & 3.088 & 0.0686 & $-4.40 \times 10^{-4}$ & 185.3 & 197.7 & 6.12 \\
${ }^{2} \Sigma^{+}$ & 2.905 & 0.0773 & $3.71 \times 10^{-4}$ & 200.5 & 201.9 & 0.698 \\
$A \frac{1}{2}$ & 2.955 & 0.0742 & & 268.1 & 281.3 & 6.60 \\
\hline \hline
\end{tabular}

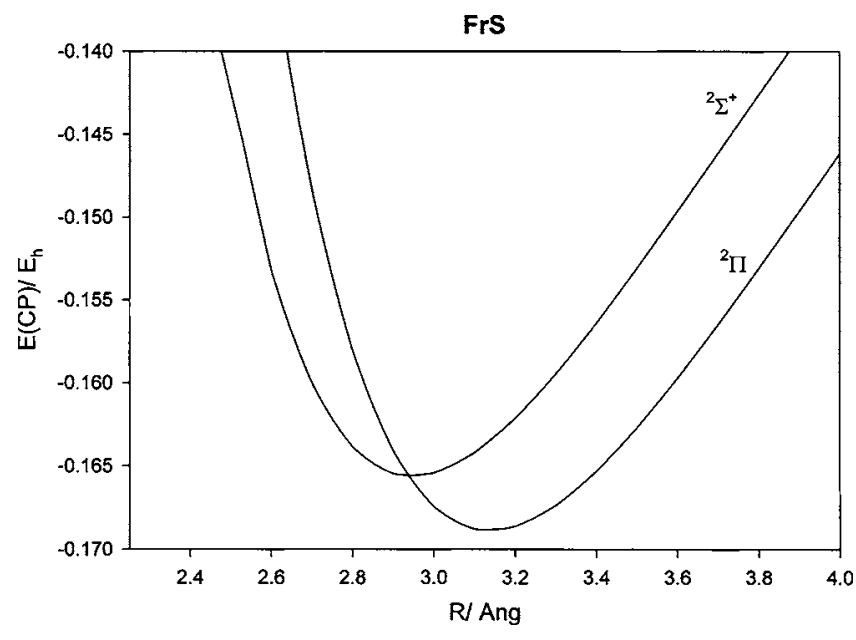

FIG. 4. Calculated potential-energy curves for FrS. The ${ }^{2} \Sigma^{+}$and ${ }^{2} \Pi$ curves are calculated in the absence of spin-orbit coupling. See text for details.

the spectroscopy of the two $X$ spin-orbit states, with an unusual negative value for the spin-rotation parameter $\alpha$ calculated for the $X \frac{1}{2}$ state.

Of note is that the ground electronic state is the ${ }^{2} \Pi$ state, in agreement with the expectation put forward in Ref. 15. Our calculated values for $T_{e}\left(T_{0}\right)$ are $654(657) \mathrm{cm}^{-1}$, indicating that the gap has narrowed significantly for CsS compared to the lighter alkali-metal sulfides (see Table III). This raises the question of whether the final member of the series, FrS, may possibly have a ${ }^{2} \Sigma^{+}$ground state.

\section{FrS}

The calculated potential-energy curves for FrS are given in Fig. 4, as may be seen, the ${ }^{2} \Pi$ state is, in fact, still the lower state. Our calculated $T_{e}\left(T_{0}\right)$ values are $714(716) \mathrm{cm}^{-1}$, i.e., a slight rise in the separation is seen compared to CsS. The calculated spectroscopic parameters are given in Table IV, but we refrain from calculating spin-orbit curves, since it is unlikely that an experimental value will be obtained for this species. We discuss the state ordering further below.

\section{E. Cations}

The alkali-metal sulfides are reasonably ionic, and so closely resemble $\mathrm{M}^{+} \mathrm{S}^{-}$close to the minimum. The lowest ionization energy (IE) is therefore expected to be largely the removal of an electron from $\mathrm{S}^{-}$, and so a fairly low IE is expected. We also calculated potential-energy curves for the lowest cationic states, $\mathrm{MS}^{+}$, obtainable by removal of an electron from each of the two lowest states of MS. Upon removal of a $\pi$ electron from the ${ }^{2} \Pi\left(\sigma^{2} \pi^{3}\right)$ state, the lowest state is expected to be the ${ }^{3} \Sigma^{-}\left(\sigma^{2} \pi^{2}\right)$ state, with associated ${ }^{1} \Delta$ and ${ }^{1} \Sigma^{+}$states expected to lie higher in energy; upon

TABLE IV. Calculated spectroscopic constants for ${ }^{223} \mathrm{Fr}^{32} \mathrm{~S}$.

\begin{tabular}{lcccccc}
\hline \hline State & $\begin{array}{c}R_{e} \\
\AA\end{array}$ & $\begin{array}{c}B_{0} \\
\left(\mathrm{~cm}^{-1}\right)\end{array}$ & $\alpha$ & $\begin{array}{c}\nu_{0-1} \\
\left(\mathrm{~cm}^{-1}\right)\end{array}$ & $\begin{array}{c}\omega_{e} \\
\left(\mathrm{~cm}^{-1}\right)\end{array}$ & $\begin{array}{c}\omega_{e} x_{e} \\
\left(\mathrm{~cm}^{-1}\right)\end{array}$ \\
\hline${ }^{2} \Pi$ & 3.137 & 0.0611 & $2.67 \times 10^{-4}$ & 186.6 & 187.8 & 0.583 \\
${ }^{2} \Sigma^{+}$ & 2.946 & 0.0693 & $3.17 \times 10^{-4}$ & 191.7 & 193.0 & 0.629 \\
\hline \hline
\end{tabular}


TABLE V. Calculated spectroscopic constants for $\mathrm{MS}^{+}$.

\begin{tabular}{lcccccrr}
\hline \hline Cation & State & $\begin{array}{c}R_{e} \\
(\AA)\end{array}$ & $\begin{array}{c}\omega_{e} \\
\left(\mathrm{~cm}^{-1}\right)\end{array}$ & $\begin{array}{c}\omega_{e} x_{e} \\
\left(\mathrm{~cm}^{-1}\right)\end{array}$ & $\begin{array}{c}\nu_{0-1} \\
\left(\mathrm{~cm}^{-1}\right)\end{array}$ & $\begin{array}{c}T_{e} \\
\left(\mathrm{~cm}^{-1}\right)\end{array}$ & $\begin{array}{c}D_{0} \\
\left(\mathrm{~cm}^{-1}\right)\end{array}$ \\
\hline $\mathrm{KS}^{+}$ & ${ }^{3} \Sigma^{-}$ & 3.313 & 118.3 & 1.56 & 115.2 & 0 & 2647 \\
& ${ }^{3} \Pi$ & 3.506 & 61.3 & 2.46 & 56.4 & 2351 & 324 \\
$\mathrm{RbS}^{+}$ & ${ }^{3} \Sigma^{-}$ & 3.528 & 92.3 & 1.16 & 90.0 & 0 & 2214 \\
& ${ }^{3} \Pi$ & 3.702 & 48.4 & 1.80 & 44.7 & 1966 & 270 \\
$\mathrm{CsS}^{+}$ & ${ }^{3} \Sigma^{-}$ & 3.728 & 79.8 & 1.01 & 77.8 & 0 & 1917 \\
& ${ }^{3} \Pi$ & 3.847 & 44.5 & 1.52 & 41.5 & 1664 & 270 \\
$\mathrm{FrS}^{+}$ & ${ }^{3} \Sigma^{-}$ & 3.791 & 75.2 & 0.93 & 73.3 & 0 & 1855 \\
& ${ }^{3} \Pi$ & 3.890 & 43.0 & 1.40 & 40.2 & 1587 & 284 \\
\hline \hline
\end{tabular}

removal of a $\sigma$ electron, a ${ }^{3} \Pi\left(\sigma^{1} \pi^{3}\right)$ state results, which is also the lowest state expected upon removal of a $\pi$ electron from the ${ }^{2} \Sigma^{+}\left(\sigma^{1} \pi^{4}\right)$ state. (We discuss these electronic states in more detail in the case of $\mathrm{NaO}$ in Ref. 28.) In all cases here, we calculated the ${ }^{3} \Sigma^{-}$state to be the lower, and so the ground electronic state of $M \mathrm{~S}^{+}$is expected to be the ${ }^{3} \Sigma^{-}$state for all of the alkali-metal monosulfides, since the ground states of $\mathrm{NaS}^{+}$and $\mathrm{LiS}^{+}$are both ${ }^{3} \Sigma^{-}$- see Ref. 16. For the monoxides the ground cationic state was also calculated to be the ${ }^{3} \Sigma^{-}$state (see Refs. 17 and 28-31). In Table V, a trend for smaller $T_{e}$ values can be seen as the group is descended, in line with the trend noted above for the neutral states. The ${ }^{3} \Sigma^{-}$state is expected to be the more strongly bound, owing to the two $\sigma$ electrons, and this is mirrored in the shorter bond length and the larger $\omega$ value for this state compared to the corresponding ${ }^{3} \Pi$ state.

\section{F. Ionization energies and heats of formation}

For each species, we also calculated the adiabatic ionization energy (AIE) and the vertical ionization energy (VIE)these are presented in Table VI, to our knowledge there are no experimental values to which to compare. We also include the corresponding values for $\mathrm{LiS}$ and $\mathrm{NaS}$ from Ref. 16. It may be seen that there is a trend for the AIE to fall off gradually between $\mathrm{LiS}$ and $\mathrm{CsS}$, but that there is a slight rise in the case of FrS-this trend is mirrored in the VIE. We interpret this as an increasing ionicity of the MS species from LiS to CsS, but with a slight increase in covalency once FrS is reached. We have noted such a trend in the corre-

TABLE VI. Calculated adiabatic ionization energy (AIE) and vertical ionization energy (VIE) for the MS species. For $M=\mathrm{K}, \mathrm{Rb}$, and $\mathrm{Cs}$, all values given correspond to the process $M \mathrm{~S}^{+}\left(X^{3} \Sigma^{-}\right)+e^{-} \leftarrow M \mathrm{~S}\left(X_{\frac{3}{2}}\right)$, and the electron is considered as being stationary. For $M=\mathrm{Li}, \mathrm{Na}$, and $\mathrm{Fr}$, no spin-orbit effects were included. No correction for zero-point vibrational energy has been made to any values.

\begin{tabular}{ccc}
\hline \hline Species & $\begin{array}{l}\text { AIE } \\
(\mathrm{eV})\end{array}$ & $\begin{array}{l}\text { VIE } \\
(\mathrm{eV})\end{array}$ \\
\hline $\mathrm{LiS}^{\mathrm{a}}$ & 7.855 & 8.019 \\
$\mathrm{NaS}^{\mathrm{a}}$ & 7.267 & 7.424 \\
$\mathrm{KS}$ & 6.700 & 6.911 \\
$\mathrm{RbS}$ & 6.525 & 6.740 \\
$\mathrm{CsS}$ & 6.424 & 6.710 \\
$\mathrm{FrS}$ & 6.437 & 6.733 \\
\hline
\end{tabular}

${ }^{\mathrm{a}}$ From Ref. 16.
TABLE VII. Calculated $\Delta H_{f}(0 \mathrm{~K})$ and $D_{0}$ for MS.

\begin{tabular}{ccc}
\hline \hline Species & $\begin{array}{c}\Delta H_{f}(0 \mathrm{~K}) \\
\left(\mathrm{kcal} \mathrm{mol}^{-1}\right)\end{array}$ & $\begin{array}{c}D_{0} \\
\left(\mathrm{kcal} \mathrm{mol}^{-1}\right)^{\mathrm{a}}\end{array}$ \\
\hline $\mathrm{KS}$ & 25.0 & 61.8 \\
$\mathrm{RbS}$ & 24.7 & 60.3 \\
$\mathrm{CsS}$ & 19.5 & 64.5 \\
$\mathrm{FrS}$ & & 59.7 \\
\hline \hline
\end{tabular}

${ }^{\mathrm{a}}$ Dissociation to neutral products.

sponding dioxides, ${ }^{32}$ oxides, ${ }^{17}$ and hydroxides ${ }^{33}$ and attributed this covalency to overlap between the $6 s$ and $6 p$ orbitals of francium, and the $p$ orbitals on oxygen; and a similar attribution is made here. We have noted that it is important to have basis functions that describe the $n=6$ orbitals of francium, and that the corresponding molecular orbitals are explicitly included in any correlation treatment.

The difference between the AIE and the VIE gives an idea of the appearance of the first bands in each photoelectron spectrum, and with a difference of $0.2-0.3 \mathrm{eV}\left(1600-2400 \mathrm{~cm}^{-1}\right)$ we expect a long vibrational progression, since the vibrational frequency of the ${ }^{3} \Sigma^{-}$state is $<120 \mathrm{~cm}^{-1}$ for $\mathrm{KS}^{+}$, and even less for the other sulfides. In addition, if one considers the $D_{0}$ values for the ${ }^{3} \Sigma^{-}$states, then it is expected that the high-energy end of each of the first photoelectron bands will be unstructured, as these energies are above the dissociation limit of each ion.

We now include the results of other factors on the calculated AIE, $T_{e}$ and $D_{e}$ for KS. The inclusion of relativistic effects led to a slight rise in the first AIE of only $93 \mathrm{~cm}^{-1}$ $\sim 0.01 \mathrm{eV})$. Calculations at the aug-cc-pV6Z level led to an AIE value of $6.722 \mathrm{eV}$, which is only $0.022 \mathrm{eV}$ higher than the aug-cc-pV5Z value (see Table VI). Extrapolating this value using the formula ${ }^{27}$ of Helgaker et al. leads to an AIE value of $6.752 \mathrm{eV}$ at the aug-cc-pVœZ level. We have also calculated the BSSE for the neutrals and the cations suggesting that the effect of the BSSE on the AIE is $43 \mathrm{~cm}^{-1}\left({ }^{3} \Sigma^{-}\right.$ $\left.{ }^{2} \Pi\right)$ and $49 \mathrm{~cm}^{-1}\left({ }^{3} \Pi-{ }^{2} \Sigma^{+}\right)$, each of which is $<0.01 \mathrm{eV}$. Based upon our previous experience with these basis sets and methods, we do not anticipate the error in the RbS-FrS ionization energies being greater than $0.05 \mathrm{eV}$. For KS, the extrapolated value is probably accurate to $0.02 \mathrm{eV}$.

The heats of formation, $\Delta H_{f}$, of $\mathrm{KS}-\mathrm{CsS}$ are calculated at $0 \mathrm{~K}$, and are given in Table VII; again, there appear to be no experimental values to which to compare. In calculating the heats of formation, we have used our CP-corrected $D_{e}$ values (for the lowest spin-orbit state) to ionic products and the JANAF (Ref. 34) $\Delta H_{f}$ values for $\mathrm{S}^{-}, \mathrm{K}^{+}, \mathrm{Rb}^{+}$, and $\mathrm{Cs}^{+}$. We also calculate dissociation energies to neutral products, again making use of the JANAF values for electron affinity (EA) of (S) and the ionization energies for the alkali metals. Looking at the dissociation energies, it is interesting to note that the values are very similar, with there being no obvious trend. The similarity can be explained by a balance between the increasing polarizability of the $\mathrm{M}^{+}$cation, together with the increased repulsion as the number of electrons increases.

For KS, we now explore the effect of other factors on the calculated dissociation energy of the ${ }^{2} \Pi$ state. First relativistic effects lead to a very slight increase in the dissociation 
energy of $35.3 \mathrm{~cm}^{-1}\left(\sim 0.1 \mathrm{kcal} \mathrm{mol}^{-1}\right)$ which is negligible. We also calculated the dissociation energy at the aug-cc-pV6Z level of theory and extrapolated to an infinite basis set size, obtaining a value of $63.3 \mathrm{kcal} \mathrm{mol}^{-1}$. Again, calculation of the BSSE was performed, and was found to be $\sim 0.2 \mathrm{kcal} \mathrm{mol}^{-1}$. We calculate an extrapolated $\Delta H_{f}(0 \mathrm{~K})$ value of $23.9 \mathrm{kcal} \mathrm{mol}^{-1}$ for KS. For RbS-FrS, based upon these considerations, plus our previous experience, it is unlikely the $\Delta H_{f}$ values are more than $2 \mathrm{kcal} \mathrm{mol}^{-1}$ in error; for $\mathrm{KS}$, the extrapolated value is unlikely to be more than $1 \mathrm{kcal} \mathrm{mol}^{-1}$ in error.

\section{CONCLUSIONS}

We have made use of high-level $a b$ initio calculations and large basis sets (ECP-based for the heavier elements) to obtain reliable potential-energy curves for $\mathrm{MS}$ and $\mathrm{MS}^{+}$. From these curves, we have calculated spectroscopic parameters, outside of the rigid-rotor harmonic-oscillator (RRHO) approximation; and for the neutrals both with and without spin-orbit coupling. We have shown that for the neutral species, the inclusion of spin-orbit coupling leads to an avoided crossing between the ${ }^{2} \Sigma^{+}$and the $\Omega=1 / 2$ component of the ${ }^{2} \Pi$ state. This crossing occurs lower down the potentialenergy curves as the metal becomes heavier, and in the case of CsS, the perturbation of the spectroscopic parameters as a result of the interaction is quite significant.

We have shown that the ground state of the MS species is, in fact, ${ }^{2} \Pi$ for all of the series, in contrast with the corresponding monoxide species, where a switch in the ground electronic state occurs at KO. Partridge et al. performed ${ }^{15}$ constrained space-orbital variation analyses for the lighter sulfides and noted that the electrostatic interaction was the predominant reason for the maintenance of a ${ }^{2} \Pi$ ground state, and it appears that this continues all of the way down the group.

The cations all have ${ }^{3} \Sigma^{-}$ground states, which is also the case for the monoxides. Ionization energies for the MS species have also been calculated, and there is some evidence for an enhancement of covalency in FrS, which goes against the trend of increasing ionicity as the group is descended. This observation is in line with our previous finding with other alkali-metal families. We also report $\Delta H_{f}$ values for the neutrals (and the corresponding values for the cations are facilely calculated from these and the AIE values).

We have also investigated the effects of relativity and infinite basis sets on the AIE and $D_{e}$ value of $\operatorname{KS}\left(X^{2} \Pi\right)$, as well as the ${ }^{2} \Sigma^{+}-{ }^{2} \Pi$ separation.

In conclusion, as part of our ongoing study of alkalimetal compounds, the whole series of alkali-metal sulfides has now been characterized and trends deduced. The chemistry and prevalence of these species are as yet unknown, although we note that Ziurys and co-workers have hypothesized that KS (Ref. 14) and NaS (Ref. 13) may be present in the late-type carbon star IRC+10216.

Of particular note is that the spectroscopy will become more and more complicated as the intersection of the ${ }^{2} \Pi$ and ${ }^{2} \Sigma^{+}$states occurs closer to the minimum of the ${ }^{2} \Pi$ curve. In fact, for FrS, a double minimum looks quite likely in the $X \frac{3}{2}$ state. Although it is highly unlikely that the FrS molecule will ever be studied, there seems to be no reason why the other alkali-metal sulfides will not be subject to experimental study in the near future. We hope this study will stimulate such experimental interest in the heavier species.

\section{ACKNOWLEDGMENTS}

The authors are grateful to the EPSRC for the award of computer time at the Rutherford Appleton Laboratories under the auspices of the Computational Chemistry Working Party (CCWP), which enabled these calculations to be performed. One of the authors (E.P.F.L.) is grateful to the Research Grant Council (RGC) of the Hong Kong Special Administration Region for support.

${ }^{1}$ C. Yamada, M. Fujitake, and E. Hirota, J. Chem. Phys. 91, 137 (1989).

${ }^{2}$ C. Yamada and E. Hirota, J. Chem. Phys. 99, 8489 (1993).

${ }^{3}$ C. Yamada, M. Fujitake, and E. Hirota, J. Chem. Phys. 90, 3033 (1989).

${ }^{4}$ C. Yamada and E. Hirota, J. Chem. Phys. 110, 2853 (1999).

${ }^{5}$ C. Yamada and E. Hirota, J. Chem. Phys. 111, 9587 (1999).

${ }^{6}$ J. N. Allison and W. A. Goddard III, J. Chem. Phys. 77, 4259 (1982).

${ }^{7}$ J. N. Allison, R. J. Cave, and W. A. Goddard III, J. Phys. Chem. 88, 1262 (1984).

${ }^{8}$ E. Hirota, Bull. Chem. Soc. Jpn. 68, 1 (1995).

${ }^{9}$ C. Yamada, S. Yamamoto, and E. Hirota (unpublished); cited as Ref. 31 in Ref. 8.

${ }^{10}$ C. W. Bauschlicher, Jr., H. Partridge, and K. G. Dyall, Chem. Phys. Lett. 199, 225 (1992).

${ }^{11}$ E. P. F. Lee, P. Soldán, and T. G. Wright, J. Chem. Phys. 117, 8241 (2002).

${ }^{12}$ M. A. Brewster and L. M. Ziurys, Chem. Phys. Lett. 349, 249 (2001).

${ }^{13}$ B. Z. Li and L. M. Ziurys, Astrophys. J. 488, L137 (1997).

${ }^{14}$ J. Xin and L. M. Ziurys, Astrophys. J. 495, L119 (1998).

${ }^{15}$ H. Partridge, S. R. Langhoff, and C. W. Bauschlicher, Jr., J. Chem. Phys. 88, 6431 (1988).

${ }^{16}$ E. P. F. Lee and T. G. Wright, Chem. Phys. Lett. 397, 194 (2004).

${ }^{17}$ E. P. F. Lee, J. Lozeille, P. Soldán, S. E. Daire, J. M. Dyke, and T. G. Wright, Phys. Chem. Chem. Phys. 3, 4863 (2001).

${ }^{18}$ EMSL Basis Set Library \{Basis sets were obtained from the Extensible Computational Chemistry Environment Basis Set Database, Version 1/29/ 01, as developed and distributed by the Molecular Science Computing Facility, Environmental and Molecular Sciences Laboratory which is part of the Pacific Northwest Laboratory, P.O. Box 999, Richland, Washington 99352, USA, and funded by the U.S. Department of Energy. The Pacific Northwest Laboratory is a multiprogram laboratory operated by Battelle Memorial Institute for the U.S. Department of Energy under Contract No. DE-AC06-76RLO 1830. (Contact David Feller or Karen Schuchardt for further information.)\}

${ }^{19}$ H. Partridge, J. Chem. Phys. 87, 6643 (1987).

${ }^{20}$ No literature reference given for rubidium on the GBSOF.

${ }^{21}$ Gaussian Basis Sets for Molecular Calculations, edited by S. Huzinaga, J. Andzelm, M. Klobukowski, E. Radzio-Andzelm, Y. Sakai, and H. Tatawaki (Elsevier, Amsterdam, 1984).

${ }^{22}$ R. J. LeRoy, LEVEL 7.2, a computer program for solving the radial Schrödinger equation for bound and quasibound levels, and calculating various expectation values and matrix elements; University of Waterloo Chemical Physics Research Program Report No. CP-555R, 2000 (unpublished).

${ }^{23}$ T. G. Wright, A. M. Ellis, and J. M. Dyke, J. Chem. Phys. 98, 2891 (1993).

${ }^{24}$ D. R. Herschbach, C. E. Kolb, D. R. Worsnop, and X. Shi, Nature (London) 356, 414 (1992).

${ }^{25}$ J. H. van Vleck, Phys. Rev. 33, 467 (1929).

${ }^{26}$ R. S. Mulliken and A. Christy, Phys. Rev. 38, 87 (1931).

${ }^{27}$ T. Helgaker, W. Klopper, H. Koch, and J. Noga, J. Chem. Phys. 106, 9639 (1997)

${ }^{28}$ P. Soldán, E. P. F. Lee, S. D. Gamblin, and T. G. Wright, Phys. Chem. Chem. Phys. 1, 4947 (1999). 
${ }^{29}$ E. P. F. Lee, P. Soldán, and T. G. Wright, Chem. Phys. Lett. 295, 354 (1998).

${ }^{30}$ P. Soldán, E. P. F. Lee, and T. G. Wright, J. Phys. Chem. A 102, 9040 (1998).

${ }^{31}$ E. P. F. Lee, P. Soldán, and T. G. Wright, Chem. Phys. Lett. 347, 481 (2001).
${ }^{32}$ E. P. F. Lee and T. G. Wright, J. Phys. Chem. A 109, 3257 (2005).

${ }^{33}$ E. P. F. Lee and T. G. Wright, J. Phys. Chem. A 107, 5233 (2003).

${ }^{34}$ M. W. Chase, Jr., NIST-JANAF Thermochemical Tables, J. Phys. Chem. Ref. Data Monog. 9 (American Chemical Society, Washington DC, 1998), 4th ed. 